\title{
Engineering of sugar transporters for improvement of xylose utilization during high-temperature alcoholic fermentation in Ogataea polymorpha yeast
}

\section{Roksolana Vasylyshyn}

Institut biologii klitini Nacional'na akademia nauk Ukraini

Olena Kurylenko

Institut biologii klitini Nacional'na akademia nauk Ukraini

Justyna Ruchala

Uniwersytet Rzeszowski

Nadiya Shevchuk

Institut biologii klitini Nacional'na akademia nauk Ukraini

Neringa Kuliesiene

Vytauto Didziojo universiteto Zemes ukio akademija

Galina Khroustalyova

Latvijas Universitate

Alexandr Rapoport

Latvijas Universitate

Rimantas Daugelavicius

Vytauto Didziojo universiteto Zemes ukio akademija

Kostyantyn Dmytruk

Institut biologii klitini Nacional'na akademia nauk Ukraini

Andriy Sibirny ( $\sim$ sibirny@yahoo.com )

Institute of Cell Biology https://orcid.org/0000-0001-8579-1566

\section{Research}

Keywords: xylose, Ogataea (Hansenula) polymorpha, xylose transporters, high-temperature alcoholic fermentation

Posted Date: February 8th, 2020

DOI: https://doi.org/10.21203/rs.2.22909/v1

License: (c) (1) This work is licensed under a Creative Commons Attribution 4.0 International License.

Read Full License 
Version of Record: A version of this preprint was published at Microbial Cell Factories on April 25th, 2020. See the published version at https://doi.org/10.1186/s12934-020-01354-9. 


\section{Abstract}

Background Xylose transport is one of the bottlenecks in the conversion of lignocellulosic biomass to ethanol. Xylose consumption by the wild-type strains of xylose-utilizing yeasts occurs once glucose is depleted resulting in a long fermentation process and overall slow and incomplete conversion of sugars liberated from lignocellulosic hydrolysates. Therefore, the engineering of endogenous transporters for the facilitation of glucose-xylose co-consumption is an important prerequisite for efficient ethanol production from lignocellulosic hydrolysates.

Results In this study, several engineering approaches formerly used for the low-affinity glucose transporters in Saccharomyces cerevisiae, were successfully applied for earlier identified transporter Hxt1 in Ogataea polymorpha to improve xylose consumption (engineering involved asparagine substitution to alanine at position 358 and replacement of $\mathrm{N}$-terminal lysine residues predicted to be the target of ubiquitination for arginine residues). Moreover, the modified versions of S. cerevisiae Hxt7 and Gal2 transporters also led to improved xylose fermentation when expressed in 0. polymorpha .

Conclusions The O. polymorpha strains with modified Hxt1 were characterized by simultaneous utilization of both glucose and xylose, in contrast to the wild-type and parental strain with elevated ethanol production from xylose. When the engineered Hxt1 transporter was introduced into constructed earlier advanced ethanol producer form xylose, the resulted strain showed further increase in ethanol accumulation during xylose fermentation. The overexpression of heterologous S. cerevisiae Gal2 had a less profound positive effects on sugars uptake rate, while overexpression of Hxt7 revealed the least impact on sugars consumption.

\section{Background}

The thermotolerant methylotrophic yeast Ogataea (Hansenula) polymorpha belongs to the native xylosefermenting yeast species with the ability to grow and ferment xylose and other sugars of lignocellulose at elevated temperatures (up to $50^{\circ} \mathrm{C}$ ). Such property of this yeast defines it as a good candidate for the development of an efficient process for simultaneous saccharification and fermentation (SSF) of lignocellulosic hydrolyzates [1]. However, to be economically viable, the main characteristics of xylose fermentation in 0. polymorpha, like ethanol yield, productivity, and co-fermentation with glucose, have to be improved.

The advanced 0. polymorpha ethanol producers from xylose were obtained by a combination of the methods of metabolic engineering and classical selection [2,3]. Although such recombinant strains were characterized by 25-30-fold improved ethanol production from xylose as compared to the wild-type strain, xylose uptake was slow and incomplete. It is known that for xylose-utilizing yeasts, glucose appeared to be the preferred sugar, being consumed first during mixed sugar fermentation $[4,5,6]$. One of the main drawbacks of the efficient conversion of sugars from the lignocellulosic hydrolysates could be an insufficient transport of xylose which additionally is competitively inhibited by glucose. One may assume 
that improvement of xylose utilization in O. polymorpha, similarly to S. cerevisiae, could be partially achieved by enhancement of the efficiency of transporters mediating the uptake of xylose. This could be achieved by expression of the specific xylose transporters from other organisms in 0. polymorpha and/or by the identification and engineering the native transporters with improved ability to transport xylose [7].

S. cerevisiae lacks a xylose-specific transport system but contains hexose transporters (Hxt) that mediate glucose uptake. Several Hxt proteins also mediate uptake of xylose albeit with lower affinity. A multiple deletion strain lacking the key transporters Hxt1-17 and Gal2 was found to be unable to grow on xylose and glucose. In this strain, the growth on xylose could be restored by the reintroduction of Hxt1, Hxt2, Hxt4, Hxt5, Hxt7, or Gal2 [8]. Different strategies were applied for the engineering of endogenous hexose transporters to construct glucose-insensitive xylose transporters by modification of crucial residues involved in glucose affinity, selectivity, and substrate translocation $[7,9,10]$. Many native pentoseassimilating species have low-affinity and high-affinity sugar transport systems for xylose uptake, both, diffusion facilitating transporters and proton symporters. More than 80 heterologous sugar transporters have already been expressed in S. cerevisiae. Among them, Sut1, Sut2, Xut1, Xut3 (Xyp33), Xut4, Xyp29 (STL12), Sut3 (Xyp37) from S. stipitis, Gxs1 and Gxf1 from Candida intermedia, At5g59250 from Arabidopsis thaliana, An29-2 and An25 from Neurospora crassa, xtrD from Aspergillus nidulans, MgT05196 from Meyerozyma guilliermondii and Xylh from Debaryomyces hansenii restored the ability of S. cerevisiae HXT null-mutant to transport xylose. Three of them were characterized to be the xylosespecific transporters: An25 and Xyp29 are xylose facilitators, with no glucose uptake activity, and Xut1 is able to transport glucose, but with lower affinity. However, the native hexose transporters showed the highest xylose uptake activities, even when compared with xylose-specific transporters, like CiGsx1 (F38139M40), SsXyp29, NcAn25, and SsXut1 [11]. Metabolic engineering strategies to reduce endogenous hexose transporter affinity for glucose or to raise the affinity for xylose could be the possible ways to improve ethanol production during mixed sugar fermentations.

In O. polymorpha wild-type strain, Hxt1 was identified as the functional hexose transporter with high similarity to low-affinity S. cerevisiae Hxt1 and Hxt3 transporters. Expression of O. polymorpha HXT1 gene was able to functionally complement growth deficiency of hexose transporter-less mutant of $\mathrm{S}$. cerevisiae, unable to grow on hexoses [12]. However, it was reported for low-affinity glucose/xylose transporters in S. cerevisiae that they are induced at high glucose concentrations following the rapid degradation in the absence of glucose [10].

In S. cerevisiae, Hxt7 seems to be the main transporter responsible for xylose uptake when xylose is the only carbon source available or during co-fermentation when glucose reaches low levels. GAL2 transcription is repressed in the presence of glucose, but when constitutively expressed, Gal2 and Hxt7 exhibited the highest xylose uptake rates among the endogenous hexose transporters $[9,11]$.

To our knowledge, till now only S. cerevisiae was used to engineer sugar transporters to achieve more efficient xylose utilization. In this study, we compared xylose uptake and ethanol production during hightemperature alcoholic fermentation in the native xylose-utilizing 0. polymorpha strains overexpressing 
engineered homologous Hxt1 transporter or heterologous Hxt7 or Gal2 transporters from S. cerevisiae. Engineering 0 . polymorpha Hxt1 transporter by substitution of asparagine to alanine residue at position 358 along with substitution of $\mathrm{N}$-terminal lysine residues predicted to be the target for ubiquitination for arginine residues had the highest positive impact on simultaneous utilization of xylose and glucose during co-fermentation of these sugars.

\section{Results}

Expression of the modified versions of Hxt1 in 0 . polymorpha hxt1 $\Delta$ mutant

Hxt1 was identified in 0. polymorpha wild-type strain as the functional hexose transporter. The amino acid sequence of O. polymorpha Hxt1 has a high homology to S. cerevisiae Hxt3 (59\% identity, $75 \%$ similarity), Hxt6 and Hxt7 (58\% identity, 73\% similarity), Hxt1 (57\% identity, 74\% similarity) (Additional file 1: Fig. S1). Overexpression of O. polymorpha HXT1 gene in the genome of hexose transporter-less mutant of $S$. cerevisiae functionally complements growth deficiency of this strain on glucose $[12,13]$.

In this study, several modified versions of Hxt1 were constructed and the effects of these modifications on xylose and glucose co-consumption during fermentation were analyzed after introduction into genome of hxt1 $\Delta$ mutant of 0 . polymorpha. The first version of Hxt1, named Hxt1_N358A, was engineered by substitution of asparagine to alanine at position 358 similarly to substitution N367A of S. cerevisiae chimeric Hxt36 transporter, which was identified as efficient for construction of glucose-insensitive xylose transporter [8].

The second version of Hxt1, named Hxt1_K, was constructed by replacement of N-terminal lysine residues predicted to be targets for ubiquitination by arginine residues. The prevention of ubiquitination is a way to reduce catabolite degradation and increase the retention of hexose transporters also in the absence of glucose in the medium [10].

The third version of Hxt1, named Hxt1_N358A_K, combined both above mentioned modifications (Additional file 2: Fig. S2, Additional file 3: Fig. S3).

The native or modified versions of Hxt1 were overexpressed in hxt1 $\Delta$ mutant and the efficiency of xylose and glucose co-utilization during high-temperature alcoholic fermentation was compared in the obtained recombinant strains. The kinetics of consumption of both sugars during mixed sugar fermentation with different ratio of xylose and glucose was studied (Fig. 1). In the wild-type strain, a sequential consumption of glucose and xylose was observed. Glucose was totally consumed from all tested media faster than xylose, but only $40 \%-60 \%$ of xylose was consumed during fermentation. The hxt $1 \Delta$ mutant was practically unable to utilize both glucose and xylose, as a result, no ethanol was produced during mutant cells incubation with these sugars (Fig. 1, Fig. 2). The overexpression of HXT1 wild-type allele in hxt1 $\Delta$ mutant restored the ability to utilize sugars in a manner similar to that in the wild-type strain. The hxt1 $\Delta /$ Hxt1_K strain also showed the bi-phase sugar consumption and at the end of fermentation, a significant amount of xylose remained unutilized. In contrast, the hxt1 $\Delta / \mathrm{Hxt1}$ _N358A and 
hxt1 $\Delta$ /Hxt1_N358A_K variants both showed co-consumption of glucose and xylose. However, at the end of fermentation, xylose still was not completely consumed (Fig. 1). Despite the difference in the dynamics of sugars utilization between transformants with HXT1 modifications, all of them were characterized by slightly improved ethanol production as compared to the wild-type strain (Fig. 2). The glucose to xylose ratio $7-3 \%$ is the closest to the ratio between these sugars in lignocellulosic hydrolysates [11, 14]. In the medium with such concentration of sugars, the overexpression of native or modified versions of HXT1 in hxt1 $\Delta$ mutant resulted in $30 \%$ increased ethanol production at 48 and $72 \mathrm{~h}$ of alcoholic fermentation as compared to the wild-type strain (Fig. 2).

To determine if the improved kinetics of xylose consumption is a result of a longer retention of the mutated Hxt1 transporter in the cytoplasmic membrane, Hxt1 and Hxt1_N358A_K versions were fused Cterminally to the fluorescent reporter GFP. These fusion proteins were expressed in the hxt1 $\Delta$ mutant. The cellular localization of the Hxt1-GFP fusion protein was assessed by fluorescence microscopy at $24 \mathrm{~h}$, 48 h, 72 h and 96 h (Fig. 3). The Hxt1_N358A_K-GFP fusion localized to the plasma membrane even after $96 \mathrm{~h}$ of cultivation in the minimal medium with xylose. In contrast, the native Hxt1 seems to be rapidly degraded from the plasma membrane when cells were grown on xylose. Thus, the mutagenesis of the three lysine residues in $\mathrm{Hxt} 1$ had a notable effect on membrane retention of Hxt1.

Expression of the engineered Hxt1, Gal2 and Hxt7 in O. polymopha

In our previous study, the advanced O. polymorpha ethanol producer from xylose was isolated by a combination of methods of metabolic engineering and classical selection. Despite increased ethanol production from xylose, the consumption of this pentose by the recombinant strain BEP cat8 $\Delta$ was insufficient and strongly inhibited by glucose [2]. The BEP cat8 $\Delta$ was used as a recipient to evaluate and compare the impact of the introduction of the overexpressed engineered endogenous Hxt1 or heterologous Hxt7 and Gal2 transporters on sugar consumption and alcoholic fermentation performance. To achieve this goal, the Hxt1_N358A_K was overexpressed into the genome of BEP cat8 $\Delta$ under control of the strong constitutive GAP promoter. The modified versions of heterologous Hxt7 and Gal2 transporters from S. cerevisiae were constructed and also overexpressed in BEP cat8 $\Delta$ strain. The Gal2 was modified by the substitution of asparagine to serine at position 376 and the Hxt7 by the substitution of the corresponding asparagine to phenylalanine at position 370 [9] (Additional file 3: Fig. S3). The expression of genes coding modified transporters was confirmed using qRT-PCR. The expression of HXT1 in BEP cat8 $\Delta$ /Hxt1_N358A_K and GAL2 in BEP cat8 $\Delta /$ Gal2_N376S strains was increased by 8 and 6 times, respectively, as compared to the parental BEP cat8 $\Delta$ strain.

The ethanol production and the kinetics of glucose and xylose consumption during high-temperature alcoholic fermentation were compared in recombinant strains carrying the modified Hxt1, Hxt7, and Gal2 transporters. The strain with modified Hxt1 was characterized by the most rapid xylose consumption among all tested transformants during xylose fermentation. After $96 \mathrm{~h}$ of fermentation, the BEP cat8 $\Delta$ /Hxt1_N358A_K strain was able to consume $49 \%$ more xylose than the parental strain, when the BEP cat8 $\Delta / \mathrm{Gal} 2 \_N 376 S$ strain consumed only $20 \%$ more xylose. The BEP cat8 $\Delta / H x t 7 \_N 370 F$ strain 
within $48 \mathrm{~h}$ of fermentation consumed xylose even slower than that of the wild-type strain, but after $96 \mathrm{~h}$ of fermentation, the uptake of xylose was improved by $20 \%$. The BEP cat8 $\Delta / \mathrm{Hxt1}$ _N358A_K strain was also characterized by the slightly increased ethanol production during xylose fermentation, reaching $20 \%$ more ethanol at $48 \mathrm{~h}$ (Fig. 4).

The uptake of glucose and xylose by strains with modified Hxt1, Hxt7 and Gal2 was also compared during fermentation in the medium with $7 \%$ of glucose and $3 \%$ of xylose. The parental BEP cat $8 \Delta$ strain was able to consume all glucose and only $30 \%$ of xylose within $48 \mathrm{~h}$. The uptake of both sugars by strain with modified $\mathrm{Hxt7}$ was the same as in the parental strain. At the same time, the strains, carrying modified Hxt1 and Gal2, consumed $73 \%$ and $62 \%$ more of xylose, respectively. However, ethanol production was increased during fermentation in all tested strains as compared to the parental strain (Fig. 5, Table 1). The ethanol yield and ethanol productivity of strains with modified Hxt1, Hxt7 and Gal2 was also higher as compared to the parental strain BEP cat8 $\triangle$. At the fermentation temperature of $45^{\circ} \mathrm{C}$ BEP cat8 $\Delta / \mathrm{Hxt} 1 \_N 358 \mathrm{~A} \_\mathrm{K}$ strain gave the highest ethanol yield $0.35 \mathrm{~g}$ ethanol/g sugar that is $30 \% \mathrm{more}$ as compared to the BEP cat8 $\Delta$ (Table 1$)$. 
Table 1

Main parameters of glucose and xylose co-fermentation (7:3) on $48 \mathrm{~h}$ at $45^{\circ} \mathrm{C}$ by the 0 . polymorpha strains tested

\begin{tabular}{|c|c|c|c|c|}
\hline Strain & $\begin{array}{l}\text { Ethanol } \\
(\mathrm{g} / \mathrm{L})\end{array}$ & $\begin{array}{l}\text { Ethanol yield } \\
\text { (g/g } \\
\text { consumed } \\
\text { substrate) }\end{array}$ & $\begin{array}{l}\text { Rate of } \\
\text { ethanol } \\
\text { synthesis } \\
\text { (g/g } \\
\text { biomass/h) }\end{array}$ & $\begin{array}{l}\text { Productivity of ethanol } \\
\text { synthesis }(\mathrm{g} / \mathrm{L} / \mathrm{h})\end{array}$ \\
\hline BEP cat8 $\Delta$ & $\begin{array}{l}18.23 \\
\pm 0.096\end{array}$ & $0.256 \pm 0.009$ & $0.165 \pm 0.004$ & $0.380 \pm 0.016$ \\
\hline BEP cat8 $\Delta /$ Hxt1_N358A_K & $\begin{array}{l}27.56 \\
\pm 0.127\end{array}$ & $0.347 \pm 0.014$ & $0.212 \pm 0.008$ & $0.574 \pm 0.029$ \\
\hline BEP cat8 $\Delta / G a l 2 \_N 376 S$ & $\begin{array}{l}22.59 \\
\pm 0.089\end{array}$ & $0.310 \pm 0.011$ & $0.188 \pm 0.004$ & $0.471 \pm 0.019$ \\
\hline BEP cat8 $\Delta / \mathrm{Hxt7}$ _N370F & $\begin{array}{l}25.44 \\
\pm 0.065\end{array}$ & $0.319 \pm 0.014$ & $0.203 \pm 0.007$ & $0.530 \pm 0.024$ \\
\hline \multicolumn{5}{|c|}{$\begin{array}{l}\text { The metabolic activity of the studied cells was assayed following the acidification of the incubation } \\
\text { medium in response to the additions of carbon substrates, xylose or glucose. We analyzed } \\
\text { recombinant strains carrying modified Hxt1, Gal2, and Hxt7 transporters comparing them to the } \\
\text { parental BEP cat } 8 \Delta \text { cells (Fig. } 6 \text { ). The cells were grown in media with a mixture of glucose and xylose } \\
\text { or xylose alone. Before the experiments, cells were starved without any carbon source. We measured } \\
\text { the capability of the starved cells to acidify the extracellular medium upon additions of D-glucose or } \\
\text { D-xylose. Table } 2 \text { summarizes results of the measurements. }\end{array}$} \\
\hline \multicolumn{5}{|c|}{$\begin{array}{l}\text { Cells grown in the mixtures of xylose and glucose, strongly acidified the external medium after the } \\
\text { addition of glucose. Cells grown at the same conditions, but after xylose addition, showed lower rates } \\
\text { of acidification, similarly to the cells grown in the medium with xylose. After the addition of xylose, the } \\
\text { most efficient acidification was performed by strain expressing Hxt1 as compared to parental BEP } \\
\text { cat8 } 8 \text { (Table 2). }\end{array}$} \\
\hline
\end{tabular}


Table 2

Acidification of the incubation medium after addition of substrates $(\mathrm{nmol} \mathrm{H} / \mathrm{min})$

\begin{tabular}{|c|c|c|c|c|}
\hline \multirow[t]{2}{*}{ Conditions } & \multicolumn{3}{|c|}{ Strains } & \multirow[b]{2}{*}{$\begin{array}{l}\text { BEP cat8 } \Delta / \\
\text { Hxt7_N370F }\end{array}$} \\
\hline & $\begin{array}{l}\text { BEP } \\
\text { cat8D }\end{array}$ & $\begin{array}{l}\text { BEP cat8 } \\
\text { Hxt1_N358A_K }\end{array}$ & $\begin{array}{l}\text { BEP } \\
\text { cat8 } 8 \text { / } \\
\text { Gal2_ } \\
\text { N376S }\end{array}$ & \\
\hline $\begin{array}{l}\text { Cells grown with glucose and xylose } \\
\text { mixture + glucose }\end{array}$ & $\begin{array}{l}0.92 \pm \\
0.06\end{array}$ & $1.0 \pm 0.13$ & $\begin{array}{l}1.10 \pm \\
0.14\end{array}$ & $0.82 \pm 0.09$ \\
\hline $\begin{array}{l}\text { Cells grown with glucose and xylose } \\
\text { mixture }+x y \text { lose }\end{array}$ & $\begin{array}{l}0.60 \pm \\
0.13\end{array}$ & $0.75 \pm 0.09$ & $\begin{array}{l}0.63 \pm \\
0.09\end{array}$ & $0.64 \pm 0.06$ \\
\hline Cells grown with xylose + xylose & $\begin{array}{l}0.50 \pm \\
0.07\end{array}$ & $0.66 \pm 0.03$ & $\begin{array}{l}0.48 \pm \\
0.09\end{array}$ & $0.54 \pm 0.12$ \\
\hline
\end{tabular}

\section{Discussion}

Xylose is the second most abundant sugar of the lignocellulose after glucose, therefore efficient conversion of xylose by microorganisms is a significant prerequisite for the development of economically feasible technology for the production of second generation ethanol. The wild-type strains of the methylotrophic thermotolerant yeast 0 . polymorpha grow well on xylose and ferment it under semianaerobic conditions, but the amounts of accumulated ethanol are very low. The reasons of this phenomenon are not known, however, as methods of molecular genetics are well developed for this organism and genome sequence is publicly available, 0 . polymorpha is considered as a promising model organism for construction of the efficient thermotolerant ethanol producer.

A combination of metabolic engineering and classical selection approaches was successfully used for improvement of parameters of xylose alcoholic fermentation in 0. polymorpha [15, 2, 3]. However, even recombinant strains with improved ethanol production up to 25-30 times were characterized by incomplete xylose utilization during fermentation. Therefore, the inefficient transport of xylose is considered to be one of the possible rate-limiting steps in xylose conversion to ethanol.

The uptake and consumption of xylose are insufficient and strongly inhibited by glucose due to the lack of xylose-specific transporters in the most of studied microorganisms. Xylose most often is transported by non-specific hexose transporters with poor affinity for xylose [4]. There have been many recent reports of improved transport and utilization of xylose in S. cerevisiae by expression of the heterologous transporters from native xylose-utilizing species $[16,17]$, as well as evolutionary or protein engineering of endogenous hexose transporters for increase of the affinity for xylose and decrease for glucose $[8,9,10]$. It was found that all of the mutated transporters had a mutation at either asparagine $376 / 370$ or threonine $219 / 213$ and it is likely that these alterations sterically prevent D-glucose but not D-xylose from 
entering the sugar-binding pocket $[9,11]$. As hexose transporter sequences are conserved, these studies could be applied to predict rational designs for rewiring new xylose transporters. In 0. polymorpha until now only Hxt1 was identified as the functional hexose transporter with high similarity to low-affinity Hxt1 and Hxt3 transporters of S. cerevisiae [12].

Here, we studied the role of $\mathrm{O}$. polymorpha engineered Hxt1 transporter in xylose utilization during hightemperature alcoholic fermentation of xylose or mixture of xylose and glucose. Moreover, several engineering approaches formerly used for low-affinity glucose transporters in S. cerevisiae were applied for modification of O. polymorpha Hxt1 transporter. Engineering O. polymorpha Hxt1 by substitution of asparagine to alanine residue at position 358 along with substitution of $\mathrm{N}$-terminal lysine residues predicted to be the target for ubiquitination for arginine residues resulted in simultaneous utilization of xylose and glucose during co-fermentation of these sugars after overexpression into genome of hxt1 $\Delta$ mutant and advanced ethanol producer BEP cat8 $\Delta$. The hxt $1 \Delta$ mutant was practically unable to utilize both glucose and xylose during co-fermentation, however was able to grow normally on $2 \%$ of glucose or xylose during cultivation under aerobic condition. During co-fermentation of $7 \%$ of glucose and $3 \%$ of xylose both sugars were almost completely utilized by strain BEP cat8 $\Delta /$ Hxt1_N358A_K expressing the modified version of Hxt1 in contrast to the parental BEP cat8 $\triangle$. However, the significant amount of xylose remained unutilized during fermentation of xylose alone by either BEP cat8 $\triangle$ or BEP cat8 $\Delta$ /Hxt1_N358A_K strains. These results suggest that Hxt1 is not crucial for xylose utilization but is involved in xylose transport in 0 . polymorpha. Therefore, the screening for other putative endogenous xylose transporters and other relevant genes seems to be a promising way to construct an efficient xylose fermenting strains.

We also compared xylose uptake and ethanol production during high-temperature alcoholic fermentation by 0 . polymorpha strains overexpressing engineered endogenous Hxt1 transporter with 0 . polymorpha strains overexpressing engineered heterologous $\mathrm{Hxt7}$ or Gal2 transporters from S. cerevisiae. The expression of the modified Hxt1 resulted in simultaneous utilization of both glucose and xylose during fermentation in the constructed 0 . polymorpha recombinant strains. The overexpression of heterologous Gal2 had a less pronounced effect on sugars uptake rate, while overexpression of Hxt7 revealed the least impact on sugars consumption.

The best reported industrial $\mathrm{S}$. cerevisiae yeast strain was able to consume both glucose and xylose with an ethanol yield of 0.46 at $32{ }^{\circ} \mathrm{C}$, however, the fermentation rate was significantly reduced at $39{ }^{\circ} \mathrm{C}[18$, 19]. The thermotolerant isolates of Pichia kudriavzevii and Candida tropicalis fermented a mixture of glucose and xylose (6:4) with ethanol yield 0.33 and $0.36 \mathrm{~g}$ ethanol/g sugar, respectively, at $42{ }^{\circ} \mathrm{C}$ [20]. Spathaspora passalidarum that is capable of co-fermenting xylose and cellobiose in the presence of glucose under oxygen-limiting conditions was able to produce $0.33 \mathrm{~g} / \mathrm{L}$ ethanol $/ \mathrm{g}$ sugar at $40^{\circ} \mathrm{C}$ during mixed sugar fermentation [21]. In this study the 0. polymorpha strains overexpressing engineered endogenous Hxt1 transporter was obtained able to produce $0.35 \mathrm{~g} / \mathrm{L}$ ethanol $/ \mathrm{g}$ sugar at $45^{\circ} \mathrm{C}$ during cofermentation of glucose and xylose. 
The overall metabolic activity of the studied cells in response to the additions of xylose or glucose was monitored by the electrochemical $\mathrm{pH}$ registration system. The cells were grown in the mixture of glucose and xylose or xylose alone. The medium acidification rate of the starved cells could be used as an indicator of the intensity of carbon catabolism upon the addition of D-glucose or D-xylose to suspensions [22]. Our data suggest that overexpression of the engineered HXT1 in 0. polymorpha cells resulted in the highest rate of extracellular acidification after addition of xylose as compared to the heterologous engineered transporters Gal2 and Hxt7.

\section{Conclusions}

In the current study, the impact of the engineered O. polymorpha Hxt1 and S. cerevisiae Gal2 and Hxt7 transporters on xylose and glucose utilization and ethanol production in 0. polymorpha were compared under conditions of high-temperature alcoholic fermentation. The expression of endogenous engineered Hxt1 in 0. polymorpha had the most pronounced effect on simultaneous sugars consumption and ethanol production during co-fermentation of glucose and xylose as compared to engineered heterologous Gal2 or Hxt7 of S. cerevisiae.

\section{Methods}

\section{Strains, media, and culture conditions}

polymorpha cells were grown on YPD (10 g/L yeast extract, $10 \mathrm{~g} / \mathrm{L}$ peptone, $20 \mathrm{~g} / \mathrm{L}$ glucose) or mineral $\left(6.7 \mathrm{~g} / \mathrm{L}\right.$ YNB without amino acids, $20 \mathrm{~g} / \mathrm{L}$ of glucose or xylose) media at $37^{\circ} \mathrm{C}$. For the NCYC495 /eu 1-1 strain, leucine $(40 \mathrm{mg} / \mathrm{L})$ was added to the medium. For selection of yeast transformants on YPD, $0.1 \mathrm{~g} / \mathrm{L}$ of nourseothricin or $0.2 \mathrm{~g} / \mathrm{L}$ of zeocin were added. Alcoholic fermentation of 0 . polymorpha cells was tested at $45^{\circ} \mathrm{C}$ as described previously [2] in the medium with $10 \%$ xylose or mixtures of glucose and xylose with ratios $7 \%: 3 \%, 5 \%: 5 \%$, or $3 \%: 7 \%$. The fermentation experiments were performed in at least triplicate to ensure the reproducibility of results. Bars in the figures indicate ranges of the standard deviation.

For electrochemical measurements, cells were taken after $24 \mathrm{~h}$ cultivation under fermentation conditions with $10 \%$ xylose or mixture of glucose and xylose (7\%: $3 \%)$. The cells were pelleted, washed with $5 \mathrm{mM}$ sodium phosphate buffer in $95 \mathrm{mM} \mathrm{NaCl}, \mathrm{pH} 7.0$ and starved with or without 2-deoxyglucose for $1 \mathrm{~h}$.

coli DH5a strain (\$80dlacZAM15, recA1, endA1, gyrA96, thi-1, hsdR17( $\left.\mathrm{rK}^{-}, \mathrm{mK}^{+}\right)$, supE44, relA1, deoR, $\triangle\left(\right.$ lacZYA-argF)U169) was used as a host for plasmid propagation. $D H 5 a$ cells were grown at $37^{\circ} \mathrm{C}$ in LB medium as described previously [23]. Transformed E. coli cells were maintained on a medium containing $100 \mathrm{mg} / \mathrm{L}$ of ampicillin.

Construction 0 . polymorpha strains with engineered endogenous Hxt1 or S. cerevisiae Hxt7 and Gal2 transporters 
polymorpha genome database (https://mycocosm.jgi.doe.gov/Hanpo2/ Hanpo2.info.html) was used for retrieval of $H X T 1$ gene sequence. The open reading frame of $H X T 1$ gene along with its own gene terminator was amplified from 0. polymorpha NCYC495 genomic DNA using primers OK161/OK162 (Additional file 4: Table S1). The native promoter of this gene was substituted by a strong constitutive promoter of glyceraldehyde-3-phosphate dehydrogenase (GAP1).

The promoter of GAP1 gene was amplified from O. polymorphaNCYC495 genomic DNA using primers OK159/OK160. The resulting fragments were fused by overlap PCR using primers OK159/OK162. The amplified fragment $(2.6 \mathrm{~kb})$ was $\mathrm{Xbal} / \mathrm{Sphl}$ digested and cloned into the corresponding sites of the pUC57/zeo vector, carrying selective marker gene conferring resistance to zeocin. The constructed plasmid was named pUC19/zeo/HXT1 (Additional file 3: Fig. S3) and used as a template for the construction of modified versions of Hxt1.

One version of Hxt1 was engineered by substitution of asparagine to alanine at position 358 to increase the specific xylose uptake rate and decrease affinity to glucose. Primers OK163/OK164 and OK165/OK162 were used for amplification of $\mathrm{N}$ - and C-fragments of HXT1 gene. The resulting fragments were fused by overlap PCR using primers OK163/OK162. The amplified fragment (1.6 kb) was Notl/Sphl digested and cloned into the corresponding sites of the pUC19/zeo/HXT1 vector instead of the native version of HXT1gene. The constructed plasmid was named pUC19/Zeo/HXT1_N358A (Additional file 3: Fig. S3).

The predicted targets of ubiquitination were identified in the sequence of $H X T 1$ gene using UbPred program software http://www.ubpred.org. The N-terminally located lysine residues at positions 8, 9 and 30 identified as potentially involved in ubiquitination were replaced for arginine residues. Primers OK159/OK167 and OK166/OK162 were used for amplification of GAP1 promoter with N-fragment and Cfragments of $H X T 1$ gene, respectively. The resulting fragments were fused by overlap PCR using primers OK159/OK162. The amplified fragment (2.6 kb) was Xbal/Sphl digested and cloned into the corresponding sites of the pUC19/zeo vector. The constructed plasmid was named pUC19/zeo/HXT1_K (Additional file 3: Fig. S3).

The modified version of $H X T 1$ with all mentioned modifications was obtained by PCR from the plasmid pUC19/zeo/HXT1_K using primers OK163/OK164 and OK165/OK162. The resulting fragments were fused by overlap PCR using primers OK163/OK162. The amplified fragment (1.6 kb) was Notl/Sphl digested and cloned into the corresponding sites of the pUC19/zeo/HXT1 vector instead of the native version of HXT1 gene. The constructed plasmid was named pUC19/zeo/HXT1_N358A_K (Additional file 3: Fig. S3).

Primers OK161/ OK216 were used for amplification of the corresponding fragment (1.6 kb) from the plasmid pUC19/zeo/HXT1_N358A_K. The resulting fragment was Notl-digested and cloned into the corresponding site of the pUC19_prGAP_NTC [24]. The constructed plasmid was named pNTC_HXT1_N358A_K (Additional file 3: Fig. S3). 
The S. cerevisiae genome database (https://www.yeastgenome.org/) was used for retrieval of HXT7 and GAL2 gene sequences. The modified version of Hxt7 was constructed by replacement of asparagine residue at position 370 for serine. Primers OK203/OK204 and OK205/OK217 were used for amplification of $\mathrm{N}$ - and $\mathrm{C}$-fragments of $H X T 7$ gene from a genomic DNA of $S$. cerevisiae BY4742. The resulting fragments were fused by overlap PCR using primers OK203/OK217. The amplified fragment (1.7 kb) was Xbal digested and cloned into the corresponding site of the pUC19_prGAP_NTC. The constructed plasmid was named pNTC_HXT7_N370A (Additional file 3: Fig. S3).

Primers OK207/OK208 and OK209/OK218 were used for amplification of $\mathrm{N}$ - and C-fragments of GAL2 gene from a genomic DNA of $S$. cerevisiae BY4742. The resulting fragments were fused by overlap PCR using primers OK207/OK218. The amplified fragment (1.7 kb) was Xbal digested and cloned into the corresponding site of the pUC19_prGAP_NTC. The constructed plasmid was named pNTC_GAL2_N376F (Additional file 3: Fig. S3).

\section{Visualization of membrane localization of Hxt1 in 0 . polymorpha by fluorescence microscopy}

The DNA fragment harboring the gene coding for the green fluorescent protein (GFP) was amplified using primers Ko819 and Ko820 from the plasmid pGFP-SLK [3]. The backbone plasmids containing HXT1 or HXT1_N358A_K was amplified with the primers Ko821/Ko822 from the plasmids pUC19/zeo/HXT1 or pUC19_prGAP_NTC_HXT1_N358A_K. Two PCR fragments were then Gibson assembled to generate the plasmids pUC19/zeo/HXT1_GFP or pUC19_prGAP_NTC_HXT1_N358A_K_GFP. These plasmids were introduced into genome of 0 . polymorpha $h x t 1 \Delta$ mutant. Transformants were selected on solid YPD medium supplemented with $0.2 \mathrm{~g} / \mathrm{L}$ of zeocin after 3 days of incubation. Selected transformants were stabilized by alternating cultivation in non-selective and selective media and examined by diagnostic PCR using primers, OK161/Ko820. The resulting strains were grown at $37^{\circ} \mathrm{C}$ in YNB medium with xylose during $96 \mathrm{~h}$ followed by microscopy analysis. Images were captured on a fluorescence microscope Axio Imager A1 (Carl Zeiss Microlmaging, Jena, Germany) coupled to a monochrome digital camera Axio Cam MRm (Carl Zeiss Microlmaging) and processed using the AxioVision 4.5 (Carl Zeiss Microlmaging) and Adobe Photoshop CC software (Adobe Systems, Mountain View, CA).

\section{Quantitative real-time PCR (qRT-PCR)}

Expression of the HXT1, HXT7 and GAL2 genes was analyzed by real-time PCR. The qRT-PCR was performed by 7500 Fast RealTime PCR System (Applied Biosystems, USA) with SG OneStep qRT-PCR kit (EURx Ltd., Gdansk, Poland) using gene-specific pairs of primers, RNA as a template, and ROX reference passive dye according to the manufacturer's instructions as described previously [2]. The pairs of primers used for qRT-PCR are listed in Additional file 4: Table S1. Sequences of the tested genes were taken from O. polymorpha genome database.

\section{Analyses}


The optical density (OD) of cell suspensions for biomass determination was measured using a «Helios- $\lambda$ » spectrophotometer at $\lambda 590$ and $\lambda 663 \mathrm{~nm}$ for 0 . polymorpha transformation [25]. Concentrations of xylose and ethanol from fermentation in the medium broth were analyzed by HPLC (PerkinElmer, Series 2000, USA) with an Aminex HPX-87H ion-exchange column (Bio-Rad, Hercules, USA). A mobile phase of $4 \mathrm{mM} \mathrm{H} 2 \mathrm{SO} 4$ was used at a flow rate $0.6 \mathrm{ml} / \mathrm{min}$ and the column temperature was $30^{\circ} \mathrm{C}$. Alternatively, concentrations of ethanol in the medium were determined using alcohol oxidase/peroxidase-based enzymatic kit "Alcotest" [26]. Experiments were performed at least twice.

\section{Measurements of the extracellular acidification}

The extracellular acidification was determined by $\mathrm{pH}$ electrode (Hanna Instruments HI1131B), measuring $\mathrm{pH}$ from 0 to 13 at -5 to $100^{\circ} \mathrm{C}$. The software NT-MDT Nova 850 and the computer program LabChart were used to monitor changes in the concentrations of hydrogen ions in the test solutions. Measurements were performed in $9 \mathrm{~mL}$ of $5 \mathrm{mM}$ sodium phosphate- $75 \mathrm{mM} \mathrm{NaCl}$ buffer, $\mathrm{pH}$ 7. At the beginning of every experiment, the signal was allowed to settle before the cells were added into the test solution. Once the signal has settled, $2 \% \mathrm{D}$-xylose or $2 \% \mathrm{D}$-glucose were added. At the end of the experiment, the known concentration of $\mathrm{NaOH}$ was added for calibration.

\section{Abbreviations}

SSF

Simultaneous Saccharification and Fermentation; qRT-PCR:quantitative reverse-transcription PCR; BEP:best ethanol producer.

\section{Declarations}

\section{Authors Contributions}

RV, OK and NS performed experiments; KD and OK designed the study; OK and RV analyzed the data and drafted the manuscript; JR performed qRT-PCR, NK analyzed the data of extracellular acidification, GK performed fluorescence microscopy, AS provided guidance and suggestions for experimental design; AR, $\mathrm{RD}, \mathrm{KD}, \mathrm{AS}$ revised the manuscript. All authors read and approved the final manuscript.

\section{Funding}

This work was supported by Ministry of Science and Education of Ukraine (grants for Joint UkrainianLithuanian research project M-98/2018 and M-74/2019), grant of the Research Council of Lithuania No SLU-18-9 and grant of National Science Centre of Poland (NCN) Opus 2016/21/B/NZ1/00280.

\section{Availability of data and materials}

The materials and datasets for the current study are available from the corresponding author on reasonable request. 
Ethics approval and consent to participate.

Not applicable.

Consent for publication.

Not applicable.

\section{Conflicts of Interest}

The authors declare no conflict of interest.

\section{References}

1.

Ryabova OB, Chmil OM, Sibirny AA. Xylose and cellobiose fermentation to ethanol by the thermotolerant methylotrophic yeast Hansenula polymorpha. FEMS Yeast Res. 2003;4(2):157-64.

2.

Ruchala J, Kurylenko 00, Soontorngun N, Dmytruk KV, Sibirny AA. Transcriptional activator Cat8 is involved in regulation of xylose alcoholic fermentation in the thermotolerant yeast Ogataea (Hansenula) polymorpha. Microb Cell Fact. 2017;16(1):36.

3.

Kurylenko 00, Ruchala J, Vasylyshyn RV, Stasyk OV, Dmytruk OV, Dmytruk KV, Sibirny AA. Peroxisomes and peroxisomal transketolase and transaldolase enzymes are essential for xylose alcoholic fermentation by the methylotrophic thermotolerant yeast, Ogataea (Hansenula) polymorpha. Biotechnol Biofuels. 2018;11:197.

4.

Saloheimo A, Rauta J, Stasyk OV, Sibirny AA, Penttilä M, Ruohonen L. Xylose transport studies with xylose-utilizing Saccharomyces cerevisiae strains expressing heterologous and homologous permeases. Appl Microbiol Biotechnol. 2007;74(5):1041-52.

5 .

Hua Y, Wang J, Zhu Y, Zhang B, Kong X, Li W, Wang D, Hong J. Release of glucose repression on xylose utilization in Kluyveromyces marxianus to enhance glucose-xylose co-utilization and xylitol production from corncob hydrolysate. Microb Cell Fact. 2019;18:24.

6.

Moreno AD, Carbone A, Pavone R, Olsson L, Geijer C. Evolutionary engineered Candida intermedia exhibits improved xylose utilization and robustness to lignocellulose-derived inhibitors and ethanol.

7.

Shin HY, Nijland JG, de Waal PP, de Jong RM, Klaassen P, Driessen AJM. An engineered cryptic Hxt11 sugar transporter facilitates glucose-xylose co-consumption in Saccharomyces cerevisiae. Biotechnol Biofuels. 2015; 8:176.

8. 
Nijland JG, Shin HY, de Jong RM, de Waal PP, Klaassen P, Driessen AJM. Engineering of an endogenous hexose transporter into a specific D-xylose transporter facilitates glucose-xylose co-consumption in Saccharomyces cerevisiae. Biotechnol Biofuels. 2014;7:168.

9.

Farwick A, Bruder S, Schadeweg V, Oreb M, Boles E. Engineering of yeast hexose transporters to transport D-xylose without inhibition by D-glucose. Proc Natl Acad Sci USA. 2014;111(14):5159-64.

10.

Nijland JG, Vos E, Shin HY, de Waal PP, Klaassen P, Driessen AJ. Improving pentose fermentation by preventing ubiquitination of hexose transporters in Saccharomyces cerevisiae. Biotechnol Biofuels. 2016;9:158.

11.

Moysés DN, Reis VC, de Almeida JR, de Moraes LM, Torres FA. Xylose Fermentation by Saccharomyces cerevisiae: Challenges and Prospects. Int J Mol Sci. 2016;17(3):207.

12.

Stasyk OG, Maidan MM, Stasyk OV, Van Dijck P, Thevelein JM, Sibirny AA. Identification of hexose transporter-like sensor HXS1 and functional hexose transporter HXT1 in the methylotrophic yeast Hansenula polymorpha. Eukaryot Cell. 2008;7(4):735-46.

13.

Wieczorke R, Krampe S, Weierstall T, Freidel K, Hollenberg CP, Boles E. Concurrent knock-out of at least 20 transporter genes is required to block uptake of hexoses in Saccharomyces cerevisiae. FEBS Lett. 1999;464(3):123-8.

14.

Hou J, Qiu C, Shen Y, Li H, Bao X. Engineering of Saccharomyces cerevisiae for the efficient co-utilization of glucose and xylose. FEMS Yeast Res. 2017;17(4).

15.

Kurylenko OO, Ruchala J, Hryniv OB, Abbas CA, Dmytruk KV, Sibirny AA. Metabolic engineering and classical selection of the methylotrophic thermotolerant yeast Hansenula polymorpha for improvement of high-temperature xylose alcoholic fermentation. Microb Cell Fact. 2014;13:122.

16.

Runquist D, Hahn-Hägerdal B, Rådström P. Comparison of heterologous xylose transporters in recombinant Saccharomyces cerevisiae. Biotechnol Biofuels. 2010;3:5.

17.

Wang C, Bao X, Li Y, Jiao C, Hou J, Zhang Q, Zhang W, Liu W, Shen Y. Cloning and characterization of heterologous transporters in Saccharomyces cerevisiae and identification of important amino acids for xylose utilization. Metab Eng. 2015;30:79-88.

18.

Demeke MM, Dietz H, Li Y, Foulquié-Moreno MR, Mutturi S, Deprez S, Den Abt T, Bonini BM, Liden G, Dumortier F, Verplaetse A, Boles E, Thevelein JM. Development of a D-xylose fermenting and inhibitor tolerant industrial Saccharomyces cerevisiae strain with high performance in lignocellulose hydrolysates using metabolic and evolutionary engineering. Biotechnol Biofuels. 2013;6(1):89. 
19.

Hou J, Qiu C, Shen Y, Li H, Bao X. Engineering of Saccharomyces cerevisiae for the efficient co-utilization of glucose and xylose. FEMS Yeast Res. 2017;17(4).

20.

Nweze JE, Ndubuisi I, Murata Y, Omae H, Ogbonna JC. Isolation and evaluation of xylose-fermenting thermotolerant yeasts for bioethanol production. Biofuels. 2019. DOI:10.1080/17597269.2018.1564480. 21.

Rodrussamee N, Sattayawat P, Yamada M. Highly efficient conversion of xylose to ethanol without glucose repression by newly isolated thermotolerant Spathaspora passalidarum CMUWF1-2. BMC Microbiol. 2018 Jul;13(1):73. 18(.

22.

Mookerjee SA, Nicholl DG, Brand MD. Determining maximum glycolytic capacity using extracellular flux measurements. PLoS One. 2016;11(3):e0152016.

23.

Sambrook J, Fritsch EF, Maniatis T. Molecular cloning: a laboratory manual. 3rd ed. New York: Cold Spring Harb. Lab; 2001.

24.

Yurkiv M, Kurylenko O, Vasylyshyn R, Dmytruk K, Fickers P, Sibirny A. Gene of the transcriptional activator MET4 is involved in regulation of glutathione biosynthesis in the methylotrophic yeast Ogataea (Hansenula) polymorpha. FEMS Yeast Res. 2018;18(2).

25.

Faber KN, Haima P, Harder W, Veenhuis M, Ab G. Highly-efcient electrotransformation of the yeast Hansenula polymorpha. Curr Genet. 1994;16:305-10.

26.

Gonchar M, Maidan M, Pavlishko H, Sibirny A. A new oxidase-peroxidase kit for ethanol assays in alcoholic beverages. Food Technol Biotechnol. 2001;39:37-42.

\section{Figures}




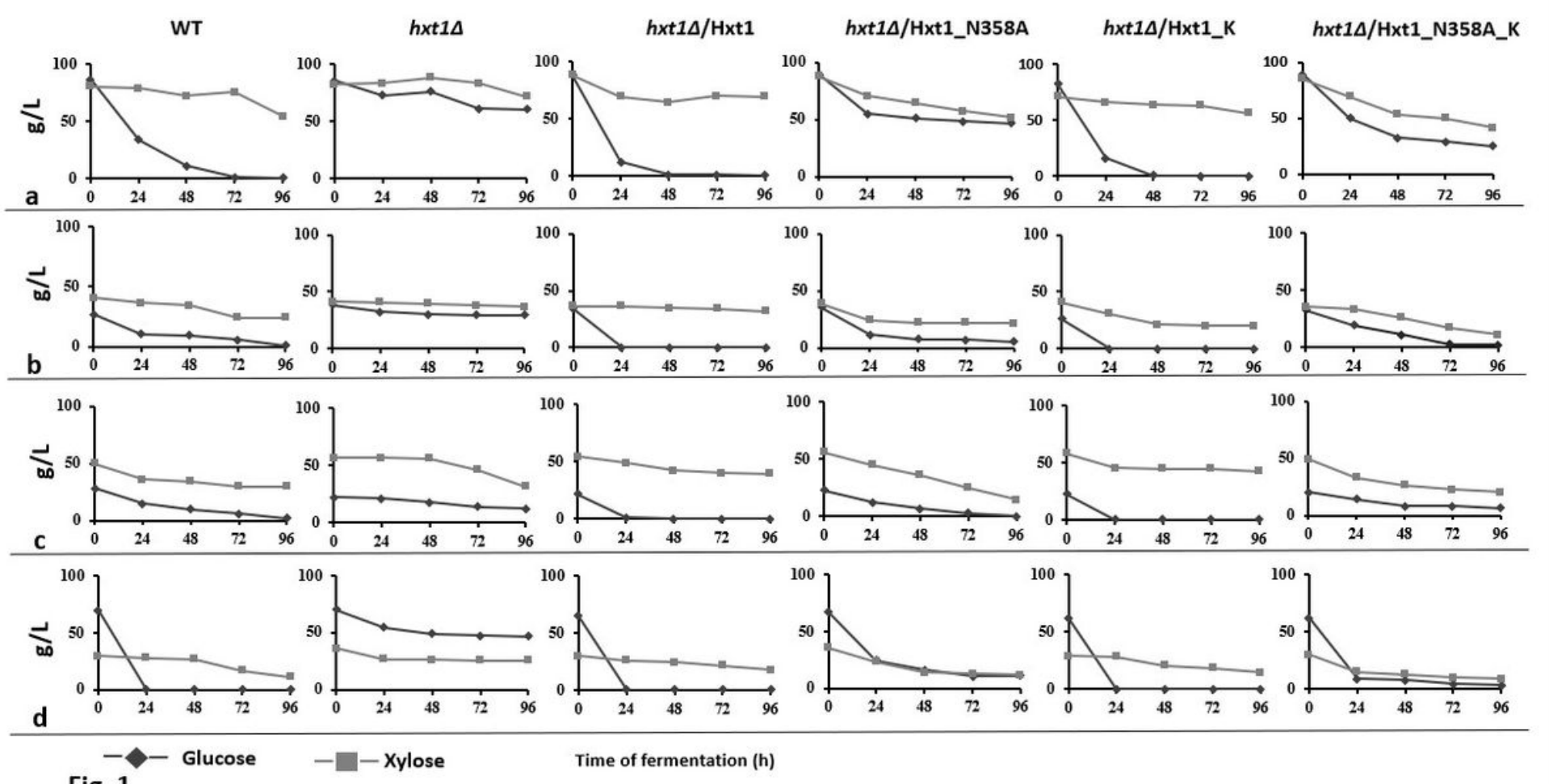

Fig. 1

\section{Figure 1}

Glucose and xylose consumption by the wild type strain (WT), hxt1 $\Delta$ mutant and obtained strains hxt1 $\Delta /$ Hxt1, hxt1 $\Delta /$ Hxt1_N358A, hxt1 $\Delta /$ Hxt1_K, hxt1 $\Delta /$ Hxt1_N358A_K during alcoholic fermentation at $45 \mathrm{C}$ in the media with different xylose to glucose ratio a) $10 \% \mathrm{xyl} / 10 \% \mathrm{glu}$ b) $5 \% \mathrm{xyl} / \mathrm{5 \%}$ glu c) $7 \% \mathrm{xyl} /$ $3 \%$ glu d) $7 \%$ glu / $3 \%$ xyl. 

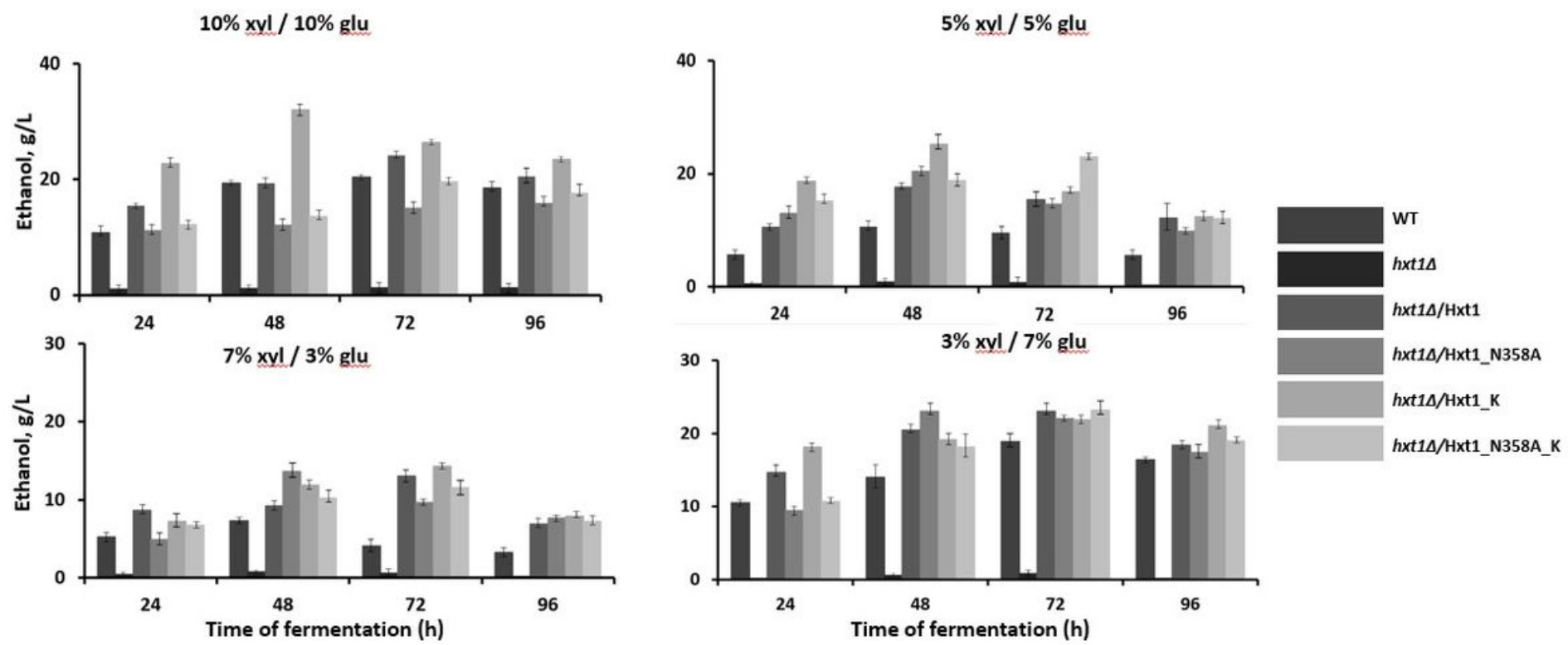

Fig. 2

\section{Figure 2}

Ethanol production by the wild-type strain (WT), hxt1 $\Delta$ mutant and obtained strains hxt1 $\Delta / \mathrm{Hxt1}$, hxt1 $\Delta /$ Hxt1_N358A, hxt1 $\Delta /$ Hxt1_K, hxt1 $\Delta /$ Hxt1_N358A_K during alcoholic fermentation at 45 oC in the media with different $h x t 1 \Delta$ xylose to glucose ratio $(x y l / g l u)$. 


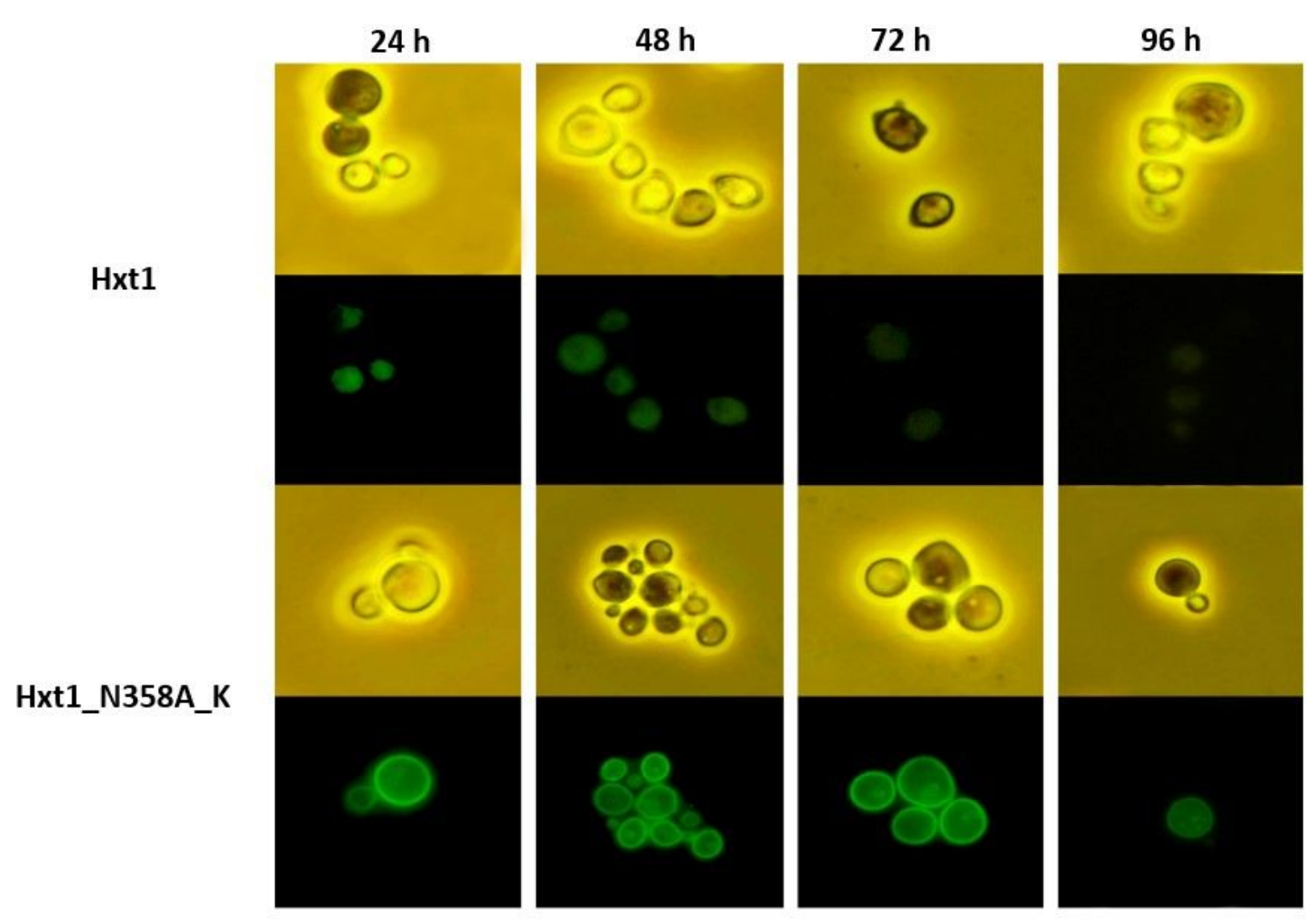

Fig. 3

\section{Figure 3}

Membrane localization of Hxt1 and Hxt1_ N358A_K fused to GFP when grown on minimal medium with $2 \%$ xylose in a 24 to $96 \mathrm{hrs}$ time range.
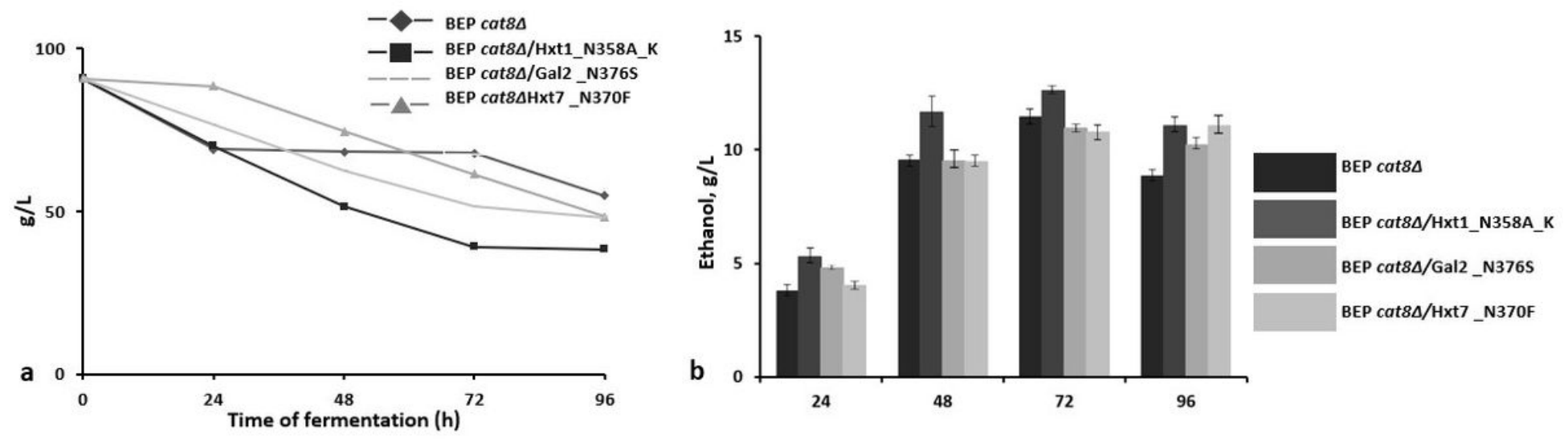

Fig. 4 
Figure 4

Xylose consumption (A) and ethanol production (B) by the parental BEP/cat8 strain and obtained strains Hxt1_N358A_K, Gal2_N376S, Hxt7_N370F during alcoholic fermentation at $45 \mathrm{C}$ in the medium with 9\% xylose.
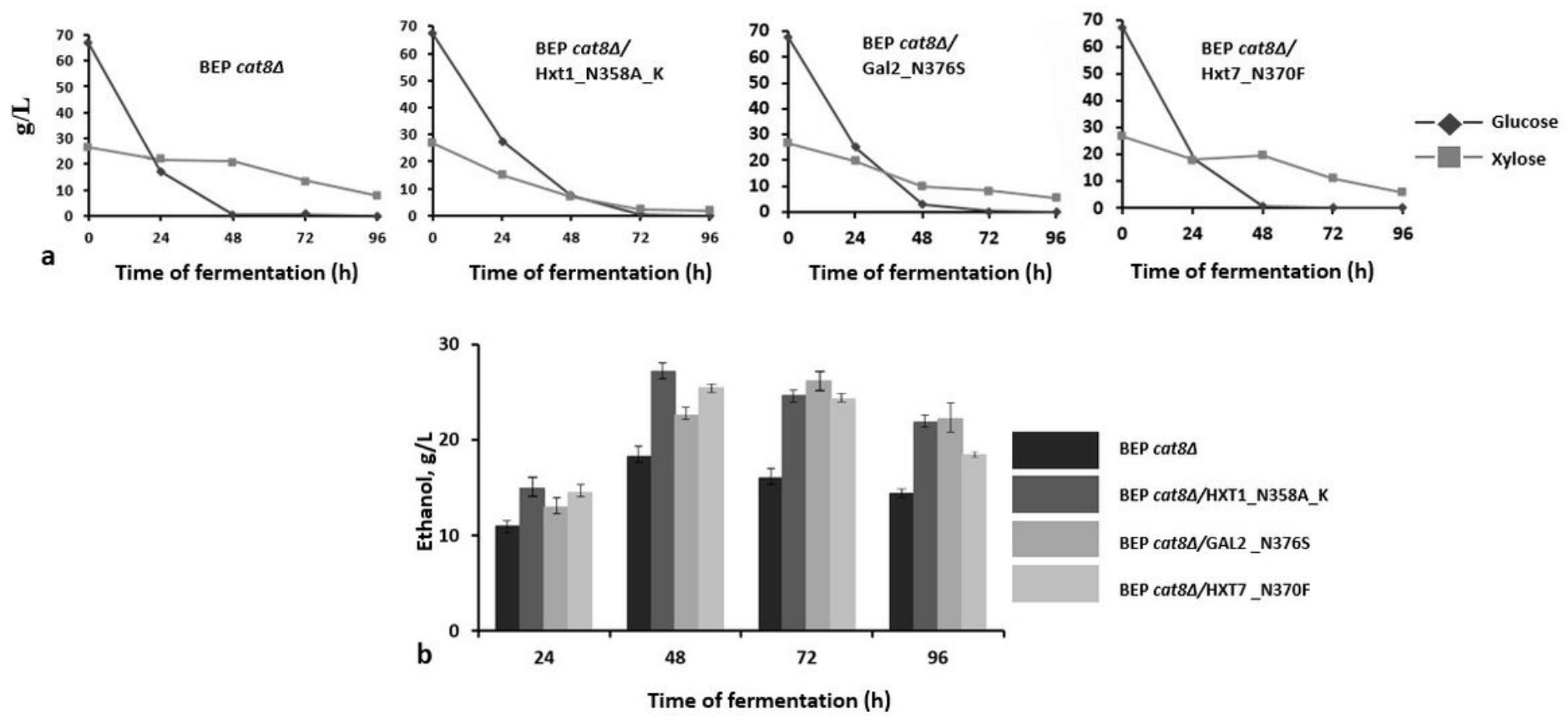

Fig. 5

\section{Figure 5}

Glucose and xylose consumption(A) and ethanol production (B) by the parental BEP/cat8 strain and obtained strains Hxt1_N358A_K, Gal2_N376S, Hxt7_N370F during alcoholic fermentation at 45 oC in the medium with $7 \%$ glucose and $3 \%$ xylose. 


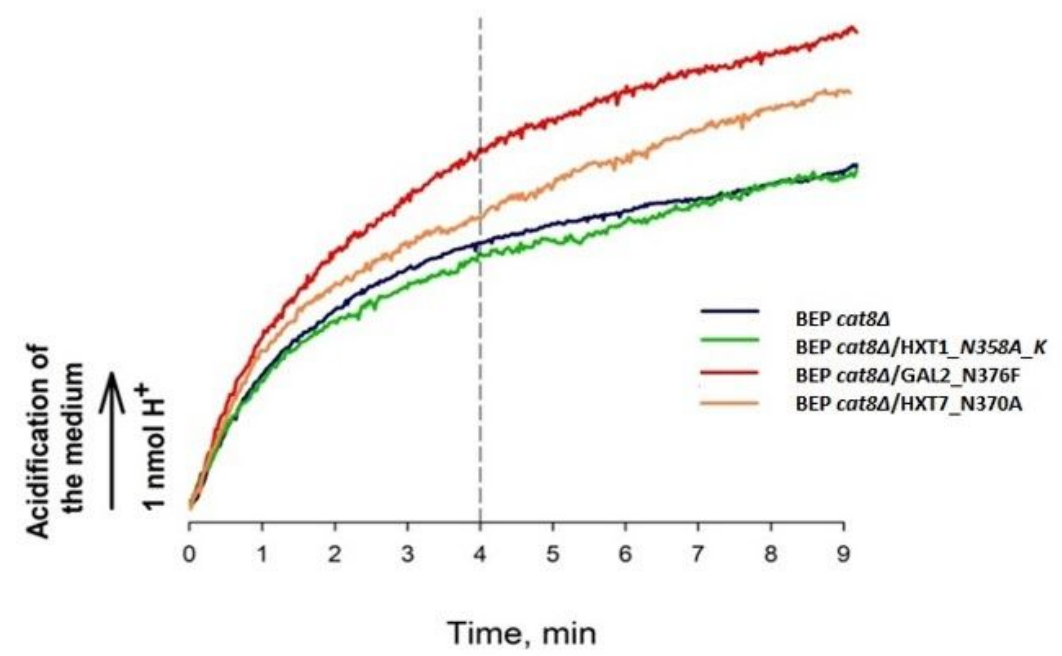

Fig. 6

\section{Figure 6}

Effect of xylose on the acidification of the incubation medium by 0 . polymorpha cells taken after $24 \mathrm{~h}$ fermentation of xylose without starvation with 2-deoxyglucose. The incubation medium contained $5 \mathrm{mM}$ sodium phosphate in solution of $95 \mathrm{mM} \mathrm{NaCl}, \mathrm{pH}$ 7. The experiments were performed at $45^{\circ} \mathrm{C}$. Yeast cells were added at 0 min to a concentration of $6 \times 107 \mathrm{cell} / \mathrm{s} / \mathrm{ml}$. The acidification rate was calculated for the first 4 min after glucose or xylose addition. The results shown are representative of three independent experiments.

\section{Supplementary Files}

This is a list of supplementary files associated with this preprint. Click to download.

- Tables1.docx

- Figurestransporterssupplementaryfinal.pptx 